\title{
Possible Atorvastatin-Induced Acute Hepatotoxicity
}

\author{
Elisa Greene*, Samantha Duncan and Kathryn P Litten \\ Pharmacy Practice Belmont University College of Pharmacy, USA
}

\begin{abstract}
Objective: To report a case of acute hepatotoxicity-induced by atorvastatin.

Practice innovation: We report the case of a 64-year-old female with a history of nonalcoholic steatohepatitis (NASH), obesity, and type II diabetes mellitus who presented with complaints of a pruritic rash, mild myalgia, icterus without nausea or vomiting, right upper quadrant (RUQ) pain, and fatigue. Lab assessment revealed elevated alkaline phosphatase of $1049 \mathrm{U} / \mathrm{L}, \mathrm{AST} 415 \mathrm{U} / \mathrm{L}$, and ALT $473 \mathrm{U} / \mathrm{L}$. Eighteen days prior to presentation, the patient was initiated on atorvastatin $40 \mathrm{mg}$ daily. Alternate causes for these extremely elevated liver enzymes were ruled out. Liver aminotransferases returned within normal range within 3 months after discontinuation of atorvastatin, but hepatic steatosis and mild fibrosis were found on a liver biopsy.
\end{abstract}

Evaluation: Statins are hepatically-cleared medications known to cause an increased risk of hepatotoxicity. Published reports characterize mild to moderate transaminase elevations with high doses of atorvastatin in high risk patients. Reports of severely elevated enzymes greater than 10 times the upper limit of normal are very infrequent, with only 4 case reports published in MEDLINE. However, details of differential diagnoses and follow-up are lacking in these reports. In our patient, a Naranjo adverse drug reaction probability scale indicates atorvastatin as probable cause to this patient's symptomatic hepatotoxicity.

Conclusion: This report exhibits one case in which a short duration of low-dose atorvastatin therapy caused symptomatic hepatosteatosis and mild fibrosis discovered by severely elevated transaminases. If liver enzymes are severely elevated, atorvastatin should be discontinued to prevent further damage.

\section{Case Description}

A 64-year-old Latina female presented to her primary care provider with the chief complaint of a 5-day history of a pruritic rash that spread to her entire body and caused difficulty sleeping. Diphenhydramine and calamine lotion tried at home was no relief. Upon questioning, she admitted to mild myalgias but denied nausea, vomiting, fever, and abdominal pain. No other household member experienced a rash, and the patient denied recent travel or use of new foods or personal products. She denied alcohol, tobacco, and illicit drug use. She reported being prescribed atorvastatin 2 months prior, but recently initiated it 3 weeks before presentation.

Past medical history was significant for fatty liver disease (NASH), obesity, type 2 diabetes mellitus, hypertension, depression, glaucoma, and hypothyroidism. Current medications included atorvastatin 40 mg daily, metformin 1,000 mg twice daily, Humalog 20 units three times daily with meals, Lantus 35-40 units every night, captopril $25 \mathrm{mg}$ daily, sertraline $50 \mathrm{mg}$ daily, brimonidine tartrate P $0.2 \%$ ophthalmic solution one drop three times daily, and levothyroxine $75 \mathrm{mcg}$ daily. Past surgical history included a cholestectomy without complications 5 years previously. An abdomen ultrasound 3 years previously revealed fatty infiltrates in the liver. Laboratory results at the time revealed a negative AMA and she was negative for hepatitis B and C. Her gamma glutamyl transpeptidase (GGT) was elevated at 130. At that time her ALT, AST, and alkaline phosphatase levels were all raised at 70, 42, and 197 respectfully. Since then her alkaline phosphatase had been fluctuating between 150-205 U/L. The patient had an 8-year history of side effects related to statins though adherence is unclear. Review of patient record revealed complaints of side effects including right upper quadrant pain and myalgias, which the patient attributed to the cholestectomy around the time of surgery, but many times could be attributed to statins. This caused her to discontinue multiple trials of low intensity lovastatin and moderate-intensity rosuvastatin as recently as one year prior to presentation.

Physical examination revealed vital signs within normal limits, including controlled blood pressure of $120 / 58 \mathrm{mmHg}$. Generalized erythematous macules were present on the abdomen, trunk, back, upper extremities, with no patches of erythema or sloughing of skin.

Laboratory testing was ordered including complete metabolic panel (CMP) and complete blood count (CBC). Hydroxyzine $25 \mathrm{mg}$ capsules three times daily for ten days and triamcinolone acetonide topical cream (TAC) $0.1 \%$ to be applied daily was prescribed on the day of presentation.

Laboratory results revealed abnormalities, including an elevated BUN of $22 \mathrm{mg} / \mathrm{dL}$ (6-20 mg/dL), RDW of $15.4 \%$ (11.5-14.5\%), alkaline phosphatase (alk phos) of $1049 \mathrm{U} / \mathrm{L}$ (41-121 U/L), AST of $415 \mathrm{U} / \mathrm{L}$ (13$35 \mathrm{U} / \mathrm{L})$, ALT of $473 \mathrm{U} / \mathrm{L}$ (7-35 U/L), potassium of $5.2 \mathrm{mmol} / \mathrm{L}$ (3.5-5.1 $\mathrm{mmol} / \mathrm{L})$ and glucose level of $155 \mathrm{mg} / \mathrm{dL}(74-106 \mathrm{mg} / \mathrm{dL})$. All other labs were within normal range. At this time the patient was instructed to discontinue atorvastatin and follow-up in clinic in 2 days.

On day 2 the pruritic rash continued despite use of hydroxyzine and TAC. The patient reported chronic mild epigastric to right upper quadrant (RUQ) pain though none worsening in the past month.

${ }^{\star}$ Correspondence to: Elisa Greene, Assistant Professor of Pharmacy Practice Belmont University College of Pharmacy 1900 Belmont Blvd Nashville, TN 37212, USA, E-mail: elisa.greene@belmont.edu

Key words: atorvastatin, acute hepatotoxicity, adverse events, statins, hepatitis

Received: October 24, 2018; Accepted: November 23, 2018; Published: November 26, 2018 
Upon examination, her sclera was white surrounding the pupil but had mild yellowing in the left eye. The patient continued to scratch at scattered papules but they did not drain or bleed. CMP was repeated and results revealed elevations similar to those seen on day 0 (Table 1). The patient was instructed to continue holding the atorvastatin and to decrease metformin dose from 2,000 mg to $1,000 \mathrm{mg}$ daily. All other medications were continued as prescribed.

On day 6 she was seen in the clinic and complained of fatigue, mild RUQ abdominal pain, and "feeling like her skin is yellowing." She reported her pruritis had mildly improved. Physical examination revealed a bilateral slight yellow discoloration of the sclera and a very mild yellow discoloration of the skin. She had a $6 \mathrm{lb}$ weight gain since first presentation 1 week prior. Her CMP laboratory results revealed continued abnormalities (Table 1). A smooth muscle antibody ELISA and anti-mitochondrial antibody titer were performed. The smooth muscle antibody returned as 17 units the next day, indicating it was negative $(<19$ units). The anti-mitochondrial antibody titer also returned negative, ruling out autoimmune disease. The patient was referred to a nearby medical center for a liver ultrasound on day 9 . The results found the liver to be of normal size with no focal hepatic abnormality or hepatomegaly, ruling out biliary obstruction. At that time, signs and symptoms were considered consistent with druginduced acute hepatitis.

The patient's CMPs were followed and her LFTs continued to decline with one elevation of unknown cause (Table 1 and Figures 1-3).

Four months after initial presentation, the patient consulted with a hepatologist at a partnering regional medical center. The patient admitted that along with prescriptions medications assessed before, she had also started taking omega-3, ginseng, and glucosamine in the same month as atorvastatin was started. She was still taking all these at presentation except had discontinued the glucosamine 2 months prior. He assessed that her signs and symptoms were consistent with acute hepatitis. At the time, AST and ALTs were only very slightly above the reference range while alkaline phosphatase was elevated at $278 \mathrm{U} / \mathrm{L}$. A MRI/MRCP was ordered to rule out post-cholecystectomy complications such as stones or biliary obstruction due to stones or sludge formation. This was found to be negative, though a few scattered background hepatic steatosis was noted. On a hepatitis virology panel she was found to be immune to hepatitis $A$, (positive HEPAIg), and negative for past or present hepatitis B and C. An immunoassay was negative, again ruling out autoimmune disease. A liver biopsy was scheduled for the following month. The biopsy revealed moderate diffuse scattered background hepatic steatosis with no focal hepatic lesions. Mild macrovesicular steatosis (approximately $20 \%$ ) and mildly increased portal fibrosis using trichome masson was found. There were no abnormal areas of enhancement or restricted

Table 1. Liver Transaminase Levels by Date

\begin{tabular}{|c|c|c|c|}
\hline Date & Alk phos (U/L) & AST (U/L) & ALT (U/L) \\
\hline $\begin{array}{c}\text { Baseline, } \\
\text { 3 years prior to } \\
\text { presentation }\end{array}$ & 166 & 23 & 21 \\
\hline Day 0 & 1049 & 415 & 473 \\
\hline Day 2 & 1034 & 344 & 510 \\
\hline Day 6 & 915 & 244 & 463 \\
\hline Day 40 & 286 & 37 & 48 \\
\hline Day 51 & 336 & 40 & 64 \\
\hline Day 94 & 239 & 28 & 36 \\
\hline $\begin{array}{c}\text { Day 142 } \\
\text { Hepatology consult }\end{array}$ & 278 & 37 & 47 \\
\hline
\end{tabular}

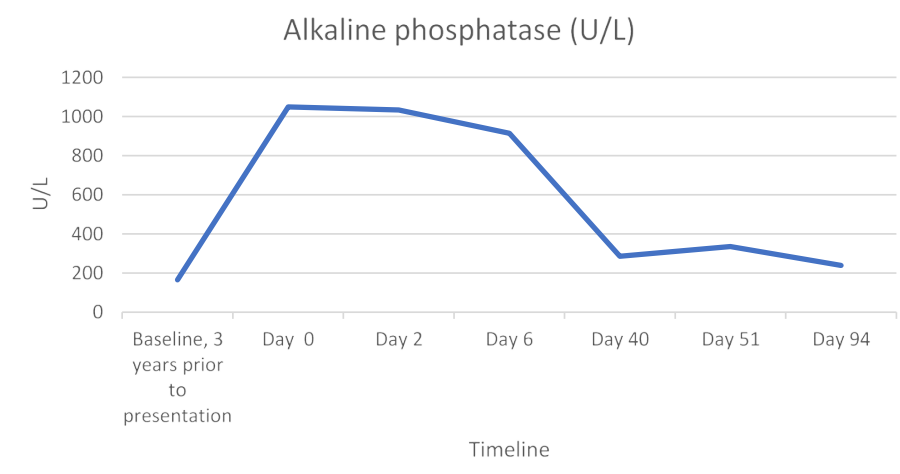

Figure 1. Alkaline Phosphatase Trend

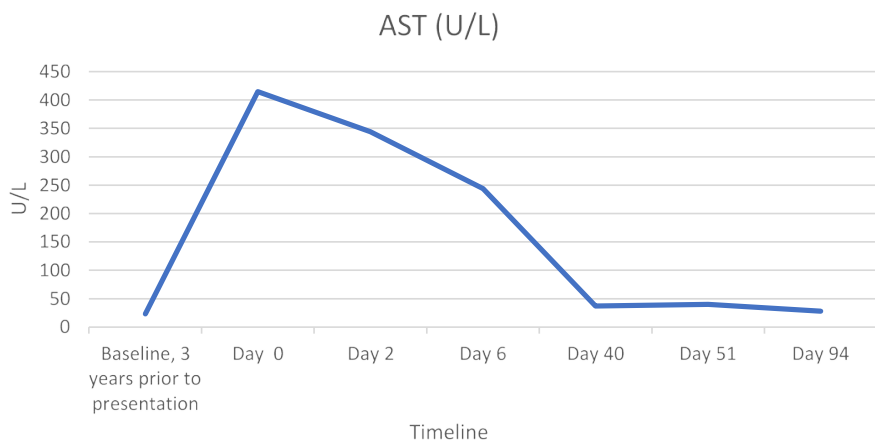

Figure 2. AST Trend

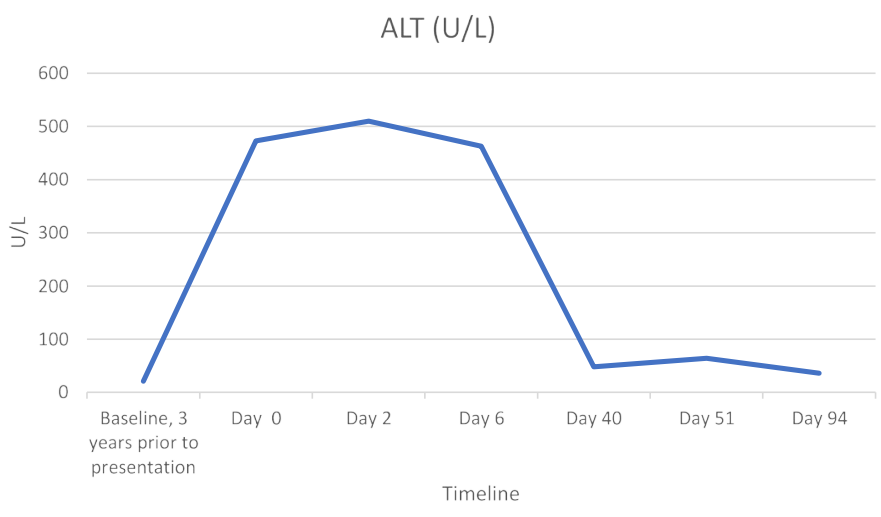

Figure 3. ALT Trend

diffusion. The gallbladder and biliary tree results were unremarkable, as were all other abdomen, heart, and musculoskeletal findings. These results were concluded to be suggestive of ongoing mild steatohepatitis. Her transaminase levels had returned to near baseline. She was seen the next month by the hepatologist to discuss plan of care. Because patients with NASH are at an increased risk for developing cardiovascular events and hyperlipidemia, the hepatologist concluded that statin therapy is not contraindicated, and is still recommended in the setting of cirrhosis due to statin-induced hepatic dysfunction being such a rare occurrence. The importance of a healthy lifestyle was stressed to prevent worsening hepatic fibrosis, cirrhosis, and hepatic decompensation.

\section{Discussion}

Atorvastatin is a widely used HMG-CoA reductase inhibitor (statin) for the treatment of dyslipidemia and is shown to be beneficial in reducing cardiovascular disease. Statins have been found to be 
generally-well tolerated. However, more severe adverse effects, such as rhabdomyalgia and hepatic failure can occur, though reports are rare [1]. At recommended doses, liver transaminase elevations of $>3 \mathrm{x}$ the upper limit of normal (ULN) occurs in $<1 \%$ of patients taking statins [2]. The presented case represents a hepatotoxicity due to atorvastatin with an increase in aminotransferase levels $>10 x U L N$ which normalized 3 months after discontinuation of the drug. Upon searching MEDLINE, only 5 similar single case reports were found, though larger studies have conflicting conclusions.

Black, et al. conducted a meta-analysis involving 4271 patients and found $0.7 \%$ had persistently elevated transaminases of $>3 x \mathrm{xLN}$, most commonly in patients taking $80 \mathrm{mg}$ daily. Only 2 patients $(0.05 \%)$ suffered from serious adverse events, including pancreatitis and cholestatic jaundice with markedly elevated transaminases; both patients recovered upon discontinuation of therapy.

The Greek Atorvastatin and Coronary Heart Disease Evaluation (GREACE) study found that patients treated with statins showed a significant improvement in AST and/or ALTs at a 3-year followup while the non-statin group had further increased. The authors concluded that statin treatment could improve LFTs in patients with mild to moderate LFT elevations [3-5].

Statins have been included in large case series of drug-induced liver injury (DILI) causes. Two large retrospective studies determining cause of liver injury, the Spanish Registry and a study in Sweden, found lipidlowering agents to be the cause of DILI in 18/461 (3\%) and 8/747(1\%) respectfully; though this included fibrates, atorvastatin, simvastatin, rosuvastatin, and Fluvastatin [6,7].

In a prospective, multicenter study by the U.S. Acute Liver Failure Study Group only 6 of the almost 2,000 patients $(0.5 \%)$ over a 10 -year period identified statins as the only agent causing acute liver failure. Only two were on atorvastatin though direct causation was nonconclusive [8].

Due to the low incidence of liver injury with statins, neither the US National Lipid Association's Safety Task Force nor the Federal Drug Administration (FDA) support the need for routine periodic liver enzyme monitoring, even in patients with asymptomatic elevated liver enzymes $[9,10]$. The FDA cited that serious liver injury with statins is "rare and unpredictable in individual patients" and routine monitoring is not effective in detecting such injury [10].

A MEDLINE search revealed 5 case reports of patients with significantly elevated transaminases and hepatic injury associated with atorvastatin therapy.

In 2002, the case of a 65-year-old woman seen for profound lower extremity weakness and dyspnea was reported. The patient had previously failed a trial of atorvastatin (time period not discussed) due to transient jaundice, but atorvastatin was reinitiated one month prior to presentation. Physical assessment revealed marked jaundice, peripheral and pulmonary edema, and muscle weakness. Acute renal failure, AST of $992 \mathrm{U} / \mathrm{L}$, ALT of $290 \mathrm{U} / \mathrm{L}$ and creatinine kinase of 8514 $\mathrm{U} / \mathrm{L}$ were also present. Although hemodialysis and intubation were initiated, the patient suffered a myocardial infarction, pancreatitis, ascites, thrombocytopenia, and deteriorated mental function before ultimately expiring from multiorgan failure. Though the author associated the cause of death with atorvastatin therapy, no differential diagnoses, comorbid conditions, or complicating factors were discussed [11].
In 2003, a report of an 83-year old man presenting with appetite loss, fatigue, discolored stools, and jaundice 2 weeks after initiation of atorvastatin $20 \mathrm{mg}$ was detailed. His ALT and ASTs were over 1300 $\mathrm{U} / \mathrm{L}$ and his alkaline phosphatase was $393 \mathrm{U} / \mathrm{L}$. The patient developed progressive encephalopathy and hepatorenal syndrome. He died 8 weeks after presentation with a highly dystrophic liver, cholestatic hepatitis, and sepsis. Though blood cultures, viral serologies, and biliary obstructions were ruled out, again differential diagnosis discussion was lacking [12].

One single-centered case analysis detailed 7 cases of atorvastatinrelated LFT elevations from 2002-2006. ALP ranged from 410-1935 $\mathrm{U} / \mathrm{L}$ and peak AST of 76-1703 U/L amongst the 7 patients. Upon discontinuation of atorvastatin, 4 patients' liver enzymes returned to normal (including the high dose patient), 1 experienced persistently abnormal LFTs, 1 experienced elevated GGT, and 1 died of acute liver failure 12 months after initiation. In this report, differential diagnoses were discussed thoroughly, and alternative causes were ruled out. They reported the probability of atorvastatin causality as 2 possible cases, 2 probable, and 1 highly probable.

In 2014 the U.S. Drug Induced Liver Injury Network (DILIN) published a report which reviewed 8 years of reported liver injuries and found only 22 of the 1,188 participants (2\%) implicated a statin. Only 2 of these 8 patients taking atorvastatin had elevated alkaline phosphatase in the thousands as our patient did with the implication of atorvastatin "probable". One of the 2 patients was taking $80 \mathrm{mg}$ daily, double the dose of our patient. Both patients' hepatotoxicity resolved [13].

In 2012, four case reports implicated a statin as the most-likely agent causing DILI including effects of jaundice, hepatitis and fatty deposits. However, each had other possible causes of liver injury including concurrent hepatotoxic medications, regular alcohol use, and a positive AMA indicating possible auto-immune etiology. The 4th case's RUCAM score was only a 5, indicating that it is only 'possibly' due to the statin $[14,15]$.

The patient described in this report was taking many other medications, however, all were ruled out as likely causes. A MEDLINE search revealed 7 cases of sertraline-induced hepatotoxicity, 2 cases of captopril-induced hepatotoxicity, and no cases of hepatotoxicity due to other medications [16-20]. The patient had been taking sertraline and captopril for over 8 years at the time of presentation and continued them throughout the entire course of treatment and follow-up. Due to the lack of temporal relationship and evidence of drug-induced hepatotoxicity, concurrent medications were ruled out.

Viral etiologies and autoimmune diseases were considered, but ruled out through multiple negative serology tests.

The patient had a history of NASH, which is defined by the National Institute of Diabetes and Digestive and Kidney Diseases as fat in the liver, along with inflammation and damage in patients who drink little to no alcohol". This can lead to cirrhosis and liver failure [21]. While NASH may have contributed to the steatosis and fibrosis, it is unlikely to have caused acute toxicity, especially in combination with a statin. The Statin Liver Safety Task Force concluded that not only was it safe to use statins in patients with NASH, but that statins may improve hepatic steatosis due to their anti-inflammatory, anti-oxidant, and antithrombotic effects $[9,22]$. The patient's NASH was diagnosed after elevated alkaline phosphatases and an ultrasound discovering fatty infiltrates 5 years prior to presentation. The patient had a past history of statin use though prior to this trial of atorvastatin, only mild symptoms and mildly elevated LFTs were noted. 
The patient's NASH was diagnosed after elevated alkaline phosphatases and an ultrasound discovering fatty infiltrates 5 years prior to presentation. Prior to starting atorvastatin, only mild symptoms and mildly elevated LFTS were noted [14].

A Naranjo algorithm was performed to estimate the probability of the atorvastatin's cause of symptoms and lab abnormalities. A score of 8 revealed a probable correlation. $(<2$ is doubtful, 2 to 4 is possible, 5 to 8 is probable, and $>9$ is definite) [20]. Therefore, after excluding all other alternatives, atorvastatin therapy is likely the cause of the steatosis and sudden, severe LFT elevations.

\section{Conclusion}

Atorvastatin and other drugs in this class are among the most frequently prescribed medications. In 2014, the CDC reported $28 \%$ of Americans over the age of 40 years used a cholesterol-lowering medication; $93 \%$ of those were statin [23]. Recent changes to the American Heart Association's guidelines estimate this number to be increased by almost 13 million Americans [24]. Though incidence of hepatotoxicity associated with atorvastatin is rare and routine monitoring of liver function tests are not recommended by the FDA, our case suggests that statins may cause lasting liver damage even after the medication is discontinued. Patients should be educated on potential side effects indicating liver abnormalities including pruritus, yellowing skin, darkening urine, fatigue, and myalgias. In light of these significant adverse effects, prescribers should strongly consider enhanced monitoring of patients who present with these symptoms upon initiation of atorvastatin [25-27].

\section{Disclaimers}

The views expressed in the submitted article are the authors' own and not an official position of the institution.

\section{Support Disclosure}

The authors have no funding or conflicts of interest to report

\section{References}

1. Atorvastatin (2016) Lexi-Drugs, Lexicomp Online. Hudson, OH: Lexicomp, Inc.

2. Farmer JA, Torre-Amione G (2000) Comparative tolerability of the HMG-CoA reductase inhibitors. Drug Saf 23: 197-213. [Crossref]

3. Black D, Farmer JA, Bakker-Arkema R. Nawroki J (1998) An overview of the clinical safety profile of atorvastatin (Lipitor), a new HMG-CoA reductase inhibitor. Arch Intern Med 158:577-85

4. Malhotra HS, Goa KL (2001) Atorvastatin: an updated review of its pharmacological properties and use in dyslipidaemia. Drugs 61: 1835-1881. [Crossref]

5. Athyros VG, Tziomalos K, Gossios TD, Griva T, Anagnostis P, et al. (2010) Safety and efficacy of long-term statin treatment for cardiovascular events in patients with coronary heart disease and abnormal liver tests in the Greek Atorvastatin and Coronary Heart Disease Evaluation (GREACE) Study: a post-hoc analysis. Lancet 376: 19161922. [Crossref]
6. Andrade RJ, Lucena MI, Fernández MC, Pelaez G, Pachkoria K, et al. (2005) Druginduced liver injury: an analysis of 461 incidences submitted to the Spanish registry over a 10-year period. Spanish Group for the Study of drug-induced liver disease. Gastroenterology 129: 512-521.

7. Björnsson E, Olsson R (2005) Outcome and prognostic markers in severe drug-induced liver disease. Hepatology 42: 481-489. [Crossref]

8. Reuben A, Koch DG, Lee WM (2010) Drug-induced acute liver failure: Results of a U.S. multicenter, prospective study. J Hepatol 52: 2065-2076.

9. Bays H, Cohen DE, Chalasani N, Harrison SA (2014) The National Lipid Association's Statin Safety Task Force (2014) An assessment by the Statin Liver Safety Task Force: 2014 update. J Clin Lipidol 8: S47-57. [Crossref]

10. FDA drug safety communication (2016) Important safety label changes to cholesterollowering statin drugs. U.S. Food and Drug Administration, FDA.

11. Sreenarasimhaiah J, Shiels P, Lisker-Melman M (2002) Multiorgan failure induced by atorvastatin. Am J Med 113: 348-349. [Crossref]

12. Perger L, Kohler M, Fattinger K, Flury R, Meier PJ, et al. (2003) Fatal liver failure with atorvastatin. J Hepatol 39: 1095-1097. [Crossref]

13. Russo MW, Hoofnagle JH, Jiezhun G (2014) Spectrum of statin hepatotoxicity: experience of the drug-induced liver injury network. Hepatology 60: 679-686.

14. RUCAM (2016) Roussel Uclaf Casuality Assessment Method () in Drug Induced Liver Injury. United Stated National Library of Medicine.

15. Bergmann OM, Kristjansson G, Jonasson JG, Björnsson ES (2012) Jaundice due to suspected statin hepatotoxicity: a case series. Dig Dis Sci 57: 1959-1964. [Crossref]

16. Deira JL, Corbacho L, Bondia A (1997) Captopril hepatotoxicity in a case of renal crisis due to systemic sclerosis. Nephrol Dial Transplant 12: 1717-1718.

17. Hagley MT, Benak RL, Hulisz DT (1992) Suspected cross-reactivity of enalapril- and captopril-induced hepatotoxicity. Ann Pharmacother 26: 780-781.

18. Tabak F, Gunduz F, Tahan V, Tabak O, Ozaras R (2009) Sertraline hepatotoxicity: Report of a case and review of literature. Dig Dis Sci 54: 1589-1591.

19. Cerda C, Bruguera M, Pares A (2013) Hepatotoxicity associated with glucosamine and chondroitin sulfate in patients with chronic liver disease. World J Gastroenterol 19: 5381-5384.

20. Naranjo CA, Busto U, Sellers EM, Sandor P, Ruiz I, et al. (1981) A method for estimating the probability of adverse drug reactions. Clin Pharmacol Ther 30: 239245. [Crossref]

21. Nonalcoholic Steatohepatitis (2016) National Institute of Diabetes and Digestive and Kidney Diseases.

22. Pastori D, Polimeni L, Baratta F, Pani A, Del Ben M, et al. (2015) The efficacy and safety of statins for the treatment of non-alcoholic fatty liver disease. Dig Liver Dis 47: 4-11. [Crossref]

23. Gu Q, Paulose-Ram R, Burt VL, Kit BK (2014) Prescription cholesterol-lowering medication use in adults aged 40 and over: United States, 2003-2012. NCHS Data Brief 177: 1-8.

24. Stone NJ, Robinson J, Lichtenstein AH (2013) 2013 ACC/AHA Guideline on the treatment of blood cholesterol to reduce atherosclerotic cardiovascular risk in adults: A report of the American College of Cardiology/American Heart Association Task Force on Practice Guidelines. J Am Coll Cardiol 63: 2889-934.

25. Clarke AT, Mills PR (2006) Atorvastatin associated liver disease. Dig Liver Dis 38 772-777. [Crossref]

26. https://labtestsonline.org/

27. Brackett CC (2010) Clarifying metformin's role and risks in liver dysfunction. $J A m$ Pharm Assoc (2003) 50: 407-410. [Crossref]

Copyright: (C2018 Greene E. This is an open-access article distributed under the terms of the Creative Commons Attribution License, which permits unrestricted use, distribution, and reproduction in any medium, provided the original author and source are credited. 